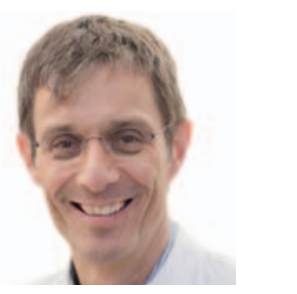

Prof. Dr. Frank Griesinger

frank.griesinger@pius-hospital.de

\title{
Netzwerke zur interdisziplinären Therapiesteuerung ermöglichen individualisierte Medizin
}

Das nicht-kleinzellige Lungenkarzinom (NSCLC) ist die häufigste zum Tode führende maligne Erkrankung beim Mann und die zweithäufigste bei der Frau. Bezogen auf alle Todesarten ist Lungenkrebs die zweithäufigste Todesursache beim Mann und die siebthäufigste bei der Frau. Führte das NSCLC noch vor 20 Jahren mit einer Cisplatin-haltigen Chemotherapie nach einem Median von 6 Monaten zum Tode, so können heute mit zielgerichteten sequentiellen Therapien bei Subgruppen mediane Überlebensraten von bis zu 50 Monaten erzielt werden.

Die Entdeckung von sogenannten driver mutations, d.h. Mutationen von EGFR, ALK und ROS, sowie die Entwicklung von zielgerichteten Medikamenten hat das NSCLC zu einer molekular definierten paradigmatischen Erkrankung werden lassen wie die CML (BCR-ABL), das Melanom (BRAF) oder gastrointestinale Stroma-Tumore (GIST). Wie bei diesen Tumoren sind inzwischen auch beim NSCLC Resistenzmechanismen zunehmend verstanden und es stehen zielgerichtete, extrem wirksame Therapien wie Osimertinib bei EGFR T790M oder Ceritinib oder demnächst Alectinib und Brigatinib bei Crizotinib-Resistenz zur Verfügung. Die Entwicklung von Methoden wie das Next Generation Sequencing (NGS) und die Liquid Biopsy (Analyse zellfreier, zirkulierender Tumor-DNA (cfctDNA)) verläuft rasant und ist bereits jetzt zur Bestimmung des molekularen Profils von histologisch gesichertem Lungenkarzinom routinemäBig im Einsatz.
Der Artikel von Reinmuth und Reck [1] (hier in deutscher Übersetzung) beschäftigt sich mit dem Einsatz von ImmuncheckpointInhibitoren, welche einen ganz neuen Therapieansatz eröffnen und bereits neben Chirurgie, Strahlentherapie, Chemotherapie und molekular-stratifizierter Therapie als 5. Säule der Krebstherapie bezeichnet werden. Obwohl das Lungenkarzinom keine besonders immunogene Erkrankung ist, sind die Substanzen wirksam und bereits in der Zweitlinientherapie etabliert. Noch in 2016 werden erste Daten zur Behandlung von Patienten in der Erstlinie veröffentlicht werden, die eine besonders hohe Expression von PDL1 aufweisen. Unter Umständen werden sich aber auch genetische Faktoren wie die durch NGS ermittelbare Mutationslast (mutational load) als Prädiktor für die Stratifizierung bei der Immuntherapie erweisen.

Die Therapiesteuerung, insbesondere der Einsatz molekular zielgerichteter Therapien bei Lungenkrebspatienten, erweist sich dann als besonders effektiv, wenn die Auswahl der Therapie auf der Kenntnis aller relevanter genetischer Tumormerkmale des Patienten beruht. Die Identifizierung dieser relevanten genetischen Alterationen und ihre Bewertung hinsichtlich ihrer therapeutischen Relevanz sind hierbei technologisch äußerst herausfordernd. Zudem stellen sich komplexe Anforderungen an Analytik, Befunderstellung und Therapieempfehlung, die nur unter Beteiligung unterschiedlicher Fachdisziplinen erfüllt werden können. Aus diesem Grunde etablieren

\section{KARGER}

Fax +497614520714

information@karger.com www.karger.com
() 2016 S. Karger GmbH, Freiburg

Accessible online at: www.karger.com/kko
Prof. Dr. med. Frank Griesinger

Klinik für Hämatologie und Onkologie, Universitätsklinik Innere Medizin-Onkologie

Pius-Hospital, Medizinischer Campus Universität Oldenburg

Georgstraße 12, 26121 Oldenburg, Deutschland 
sich zunehmend Netzwerke wie z.B. das Netzwerk Nord-Ost-West Lunge (NOWEL), dessen Sprecher der Autor ist. Dieses Netzwerk schließt die Vertreter der relevanten Fachdisziplinen (Onkologie, Pathologie, Pneumologie) zusammen, um in der jeweiligen Region die optimale Versorgung von Patienten mit Lungenkrebs zu gewährleisten. Optimalerweise sollten sich die Netzwerke durch eine interdisziplinäre und sektorenübergreifende Ausrichtung auszeichnen und die medizinische Versorgung sektorenunabhängig abbilden. Die durch Netzwerke wie NOWEL garantierte Versorgung sollte eine zentrale genetische Analytik auf höchstem technologischem Niveau mit externer Qualitätskontrolle beinhalten sowie eine fortlaufend an den neuesten Stand der Forschung angepasste Befunderhebung und eine Therapieempfehlung, die sich an der individuellen Situation des Patienten orientiert. Dies sollte am besten in einem «molekularen» Tumorboard unter Beteiligung der o.g. Fachdisziplinen erfolgen, eine Dokumentation des Outcomes der im Netzwerk behandelten Patienten sollte ebenfalls implementiert sein. Idealerweise sollten im Netzwerk Studien für Patienten mit seltenen Alterationen angeboten werden. Auf diese Weise kann sichergestellt werden, dass Neuerungen der individualisierten Medizin einer regional definierten Population von Lungenkrebspatienten unmittelbar zugutekommen und unter Berücksichtigung aller qualitätsrelevanten Standards hinsichtlich ihres Nutzens analysiert werden.

Eine interessante Lektüre wünscht Ihnen

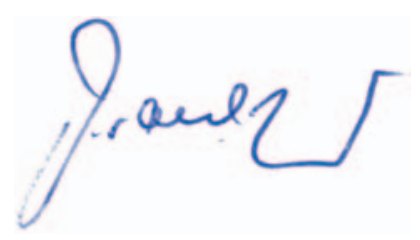

\section{Referenzen}

1 Reinmuth N, Reck M: Immunotherapy for lung cancer. Oncol Res Treat 2016;39:360-368. 